Supplementary Information for:

\title{
Probing Matrix Effects on the Heterogeneous Photochemistry of the Neonicotinoid Pesticides Dinotefuran and Nitenpyram
}

Andrea Rohrbacher, Michael J. Ezell, Véronique Perraud, and Barbara J. Finlayson-Pitts

\author{
Department of Chemistry \\ University of California Irvine \\ Irvine, CA 92697-2025
}

ACS Earth and Space Science

Submitted March 3, 2021

Keywords: ATR-FTIR, KBr pellet, nitrous oxide, nitroguanidine, quantum yield, emerging contaminants, insecticides 
A. NN/KBr pellet surfaces via SEM-EDS
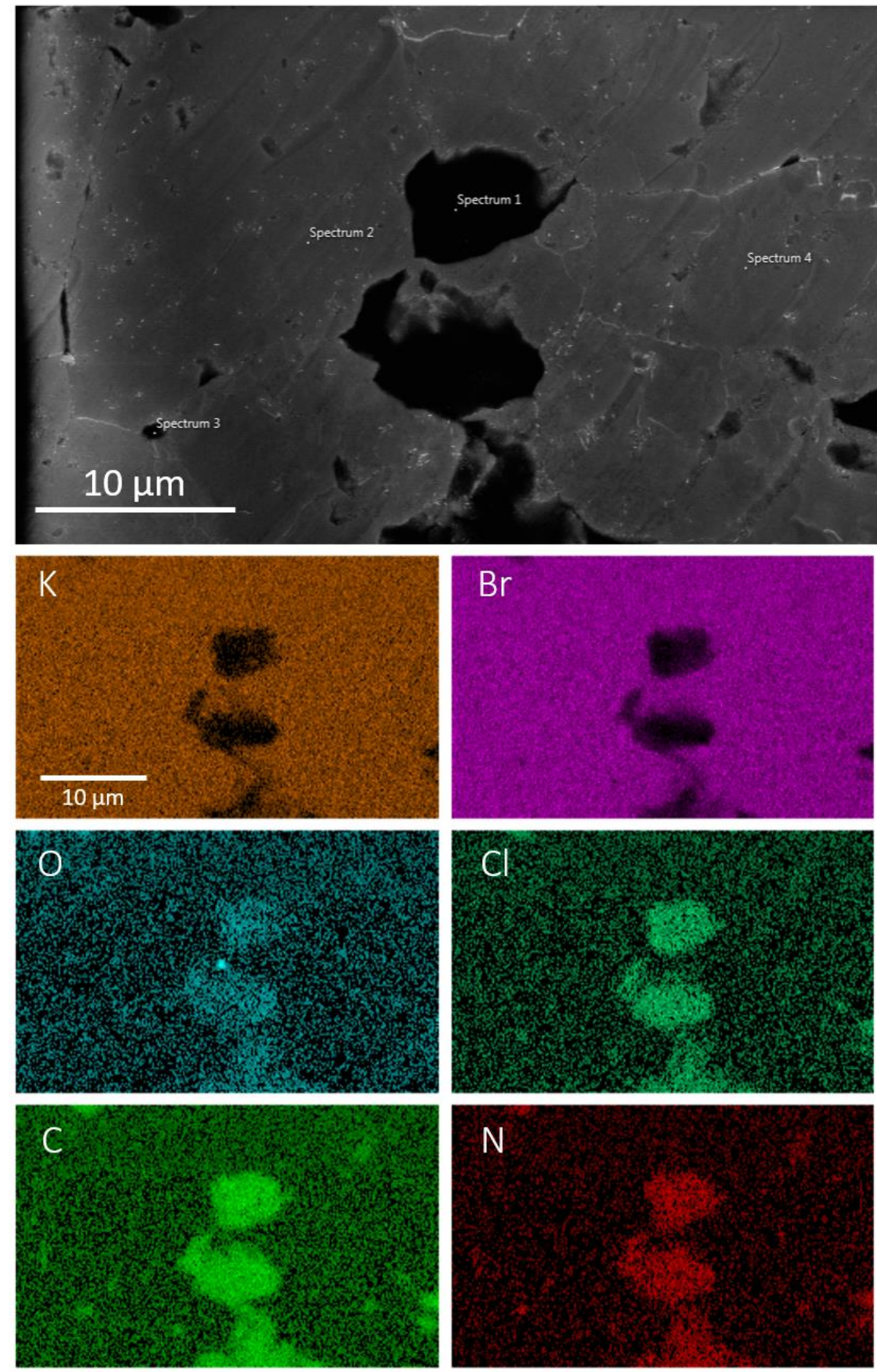

Figure S1: SEM-EDS image of 5\% imidacloprid (IMD)/KBr pellet surface mounted on carbon tape showing distribution of $\mathrm{K}, \mathrm{Br}, \mathrm{O}, \mathrm{Cl}, \mathrm{C}$, and $\mathrm{N}$. Colored areas show the presence of individual elements. IMD was used because of the presence of a Cl atom which has a strong SEM-EDS signal and facilitates detection. 


\section{B. FTIR cell details}

(a) FTIR Cell

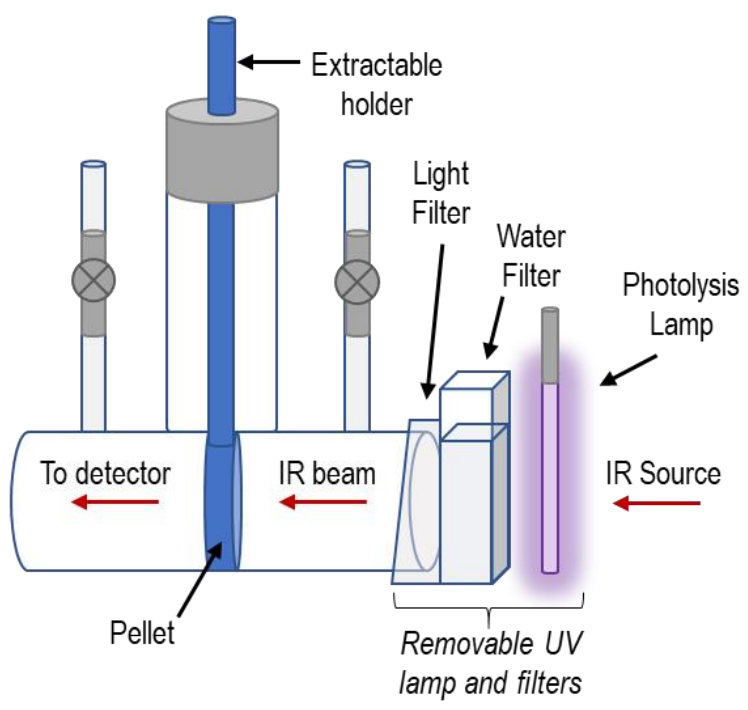

(b) $\mathrm{KBr}$ pellet
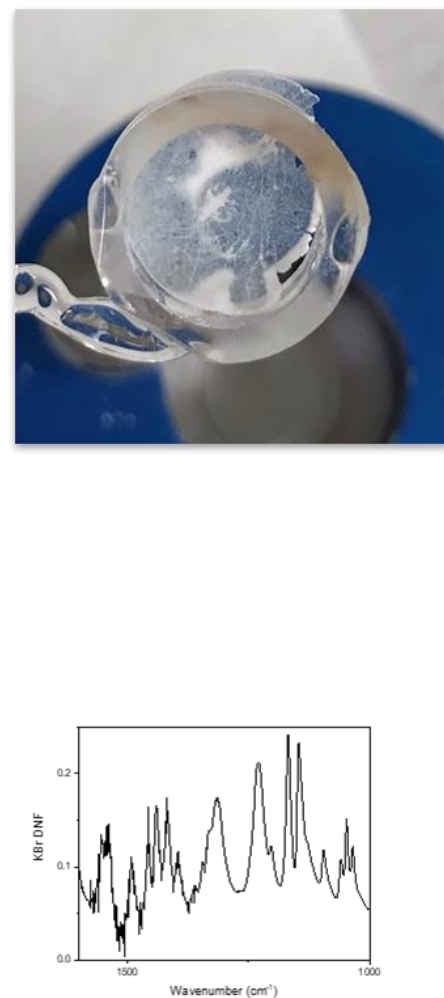

Spectral Subtraction Solid Only

Figure S2: (a) Schematic of the reaction cell. Samples were attached to a circular sample holder connected to a retractable rod and placed in a custom-built glass FTIR cell with $\mathrm{CaF}_{2}$ windows. Light below $290 \mathrm{~nm}$ was removed using a glass filter and a water-filled cuvette was used to absorb heat from the lamp. The lamp, water compartment, and light filter were removed to probe the NN/KBr pellet with the IR beam. (b) A photo of a typical NN/KBr sample pellet. (c) The combined gas and solid spectra are measured with the rod in the down position and the gas phase only in the rod up position. Spectra of the solid pellet only were obtained by subtracting the gas phase spectrum from the combined gas and solid spectrum. 


\section{Lamp emission spectra}

The emission spectra for the three lamps used in this study were measured using an Ocean Optics spectrometer (model HR 4000 CG-UV-NIR) and are shown in Fig. S3.

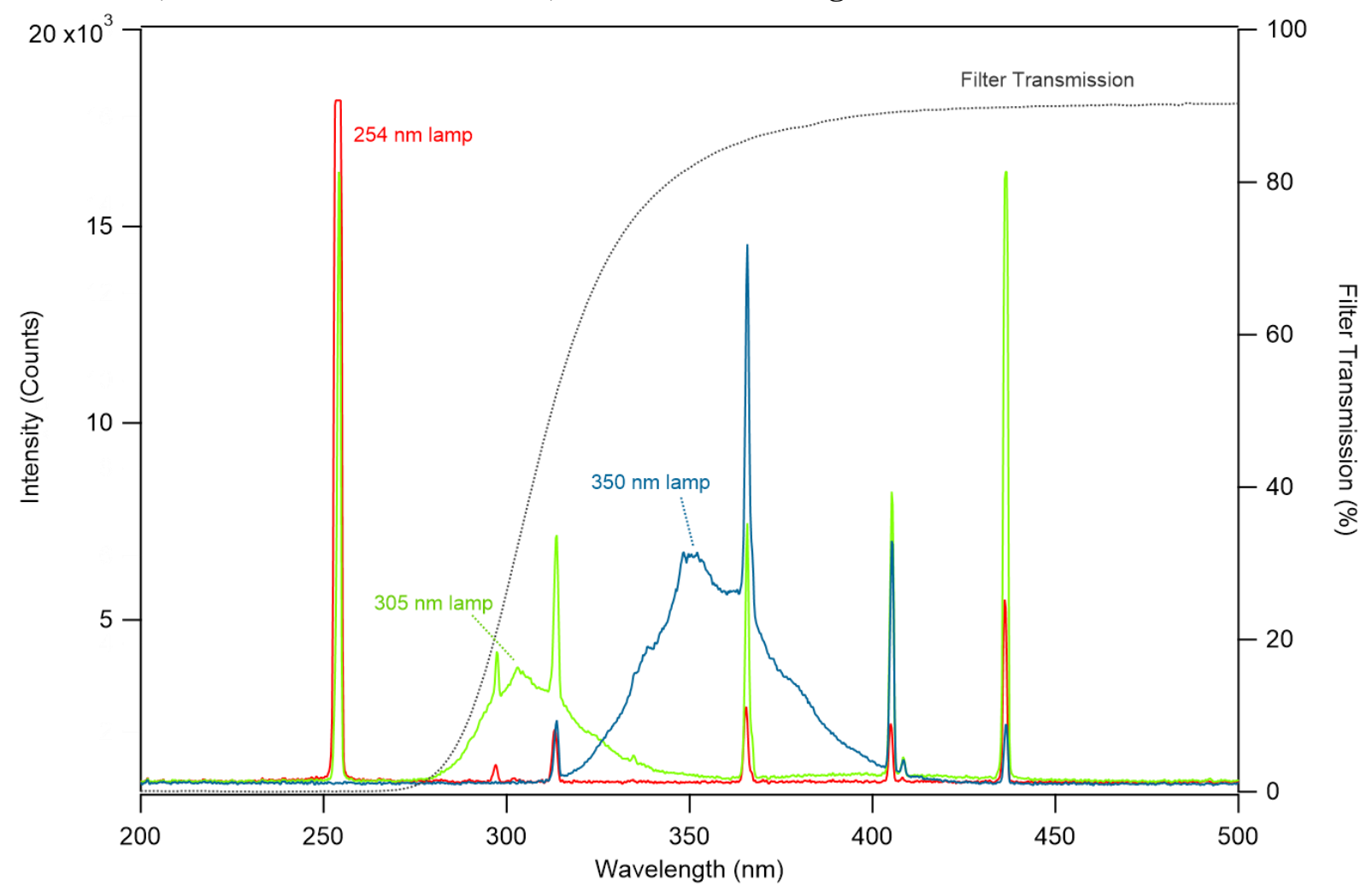

Figure S3: Relative emission spectra of UV lamps

The lamp designated $254 \mathrm{~nm}$ is a low pressure mercury lamp, which has its most prominent line at 254 $\mathrm{nm}$. The lamp designated $305 \mathrm{~nm}$ is a broadband organophosphor coated mercury lamp. Mercury lines can be seen at 254, 313 and $365 \mathrm{~nm}$, and these mercury lines excite the organophosphor coating resulting in the broadband emission centered around $305 \mathrm{~nm}$. The $350 \mathrm{~nm}$ broadband lamp is similarly an organophosphor coated mercury lamp with broadband emission spectra centered around $350 \mathrm{~nm}$. All lamps were made with ozone-free quartz which filters the majority of light below $242 \mathrm{~nm}$. A Pyrex slide was used to filter light below $290 \mathrm{~nm}$ for use with the $305 \mathrm{~nm}$ and $350 \mathrm{~nm}$ broadband lamps for actinic region-relevant measurements. 


\section{HRMS sample analysis details}

Accurate mass measurements were performed on a UPLC-DAD-HRMS platform (ThermoScientific) equipped with a Vanquish Horizon UPLC system (including a binary LC pump, an autosampler, a column manager and a diode array detector) coupled to a high-resolution Q Exactive Plus orbitrap mass spectrometer. Sample injection volume was $10 \mu \mathrm{L}$. A Luna Omega 1.6 $\mu \mathrm{m}$ Polar C18 $150 \times$ $2.1 \mathrm{~mm}$ column (Phenomenex) fitted with a SecurityGuard ULTRA cartridge (porous polar C18, $2.1 \mathrm{~mm}$; Phenomenex) maintained at $30{ }^{\circ} \mathrm{C}$ was used for the separation.

The mobile phase combination was (A) $0.1 \%$ formic acid (Fisher Chemical) in HPLC grade water (Fisher Chemical) and (B) $0.1 \%$ formic acid in HPLC grade acetonitrile (Fisher Chemical). The eluent gradient was as follows: 0-3 min hold at 5\% B, 3-14 min linear gradient to 95\% B, 14-16 min hold at 95\% $\mathrm{B}, 16-22$ min linear gradient back to $5 \% \mathrm{~B}$ with a flow rate of $300 \mu \mathrm{L} \mathrm{min}{ }^{-1}$.

UV-vis absorption spectra were acquired using the diode array detector (DAD) over the full 190$680 \mathrm{~nm}$ wavelength range. Exiting the DAD, the samples were introduced into the mass spectrometer using a heated electrospray source (HESI; ThermoScientific). Source conditions were as follows: capillary voltage, $3.50 \mathrm{kV}$; capillary temperature, $325^{\circ} \mathrm{C}$; sheath gas flow rate, 50; auxiliary gas flow rate, 10; sweep gas flow rate, 1 ; S-lens RF level 50; auxiliary gas heater temp, $300{ }^{\circ} \mathrm{C}$.

The analysis was done in ESI(+) ion mode using a full scan data-dependent MS/MS (FS-ddMS2) technique. In this approach, full MS scans were recorded over the m/z 50-750 mass range with a resolution of 140,000 and an automatic gain control (AGC) target of $10^{6}$ (max. injection time (IT) $=100$ $\mathrm{ms}$ ) while MS/MS scans were recorded for the top 3 most abundant ions from the adjacent full MS scan (resolution, 17,500; AGC target, $5 \times 10^{4}$; max. IT, $50 \mathrm{~ms}$ ) using normalized collision energies (NCE) of 10, 30 and 50. The resulting MS/MS spectra are the sum of all the fragment ions collected at all 3 collision energies.

The mass spectrometer was calibrated weekly for mass accuracy using the ThermoScientific Pierce LTQ Velos ESI positive calibration mixture containing butylamine, caffeine, MRFA and Ultramark-1621. The data were acquired using Xcalibur 4.2 software (ThermoScientific) and processed with Freestyle v1.6. 
E. Spectra of desnitro $\left(\mathrm{DN}_{\mathrm{IMD}}\right)$ and urea $\left(\mathrm{UR}_{\mathrm{IMD}}\right)$ products of IMD photolysis in $\mathrm{KBr}$ pellet

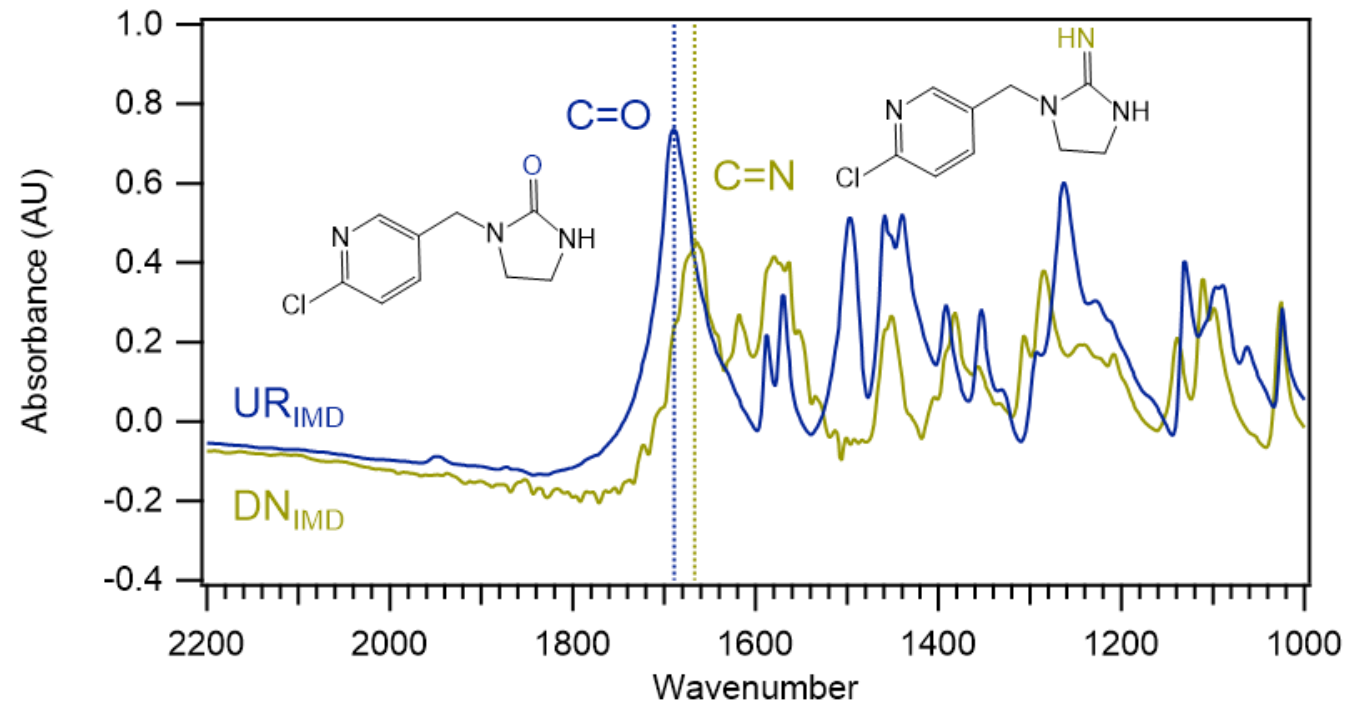

Figure S4: $4.8 \% U R_{I M D} / \mathrm{KBr}$ and $4.8 \% \mathrm{DN} I M D / \mathrm{KBr}$ reference spectra. While the molar concentrations are almost identical, the $C=O$ stretch shows a significantly stronger absorbance than the $C=N$ stretch. 
F. MS/MS analysis and proposed fragmentation pathways

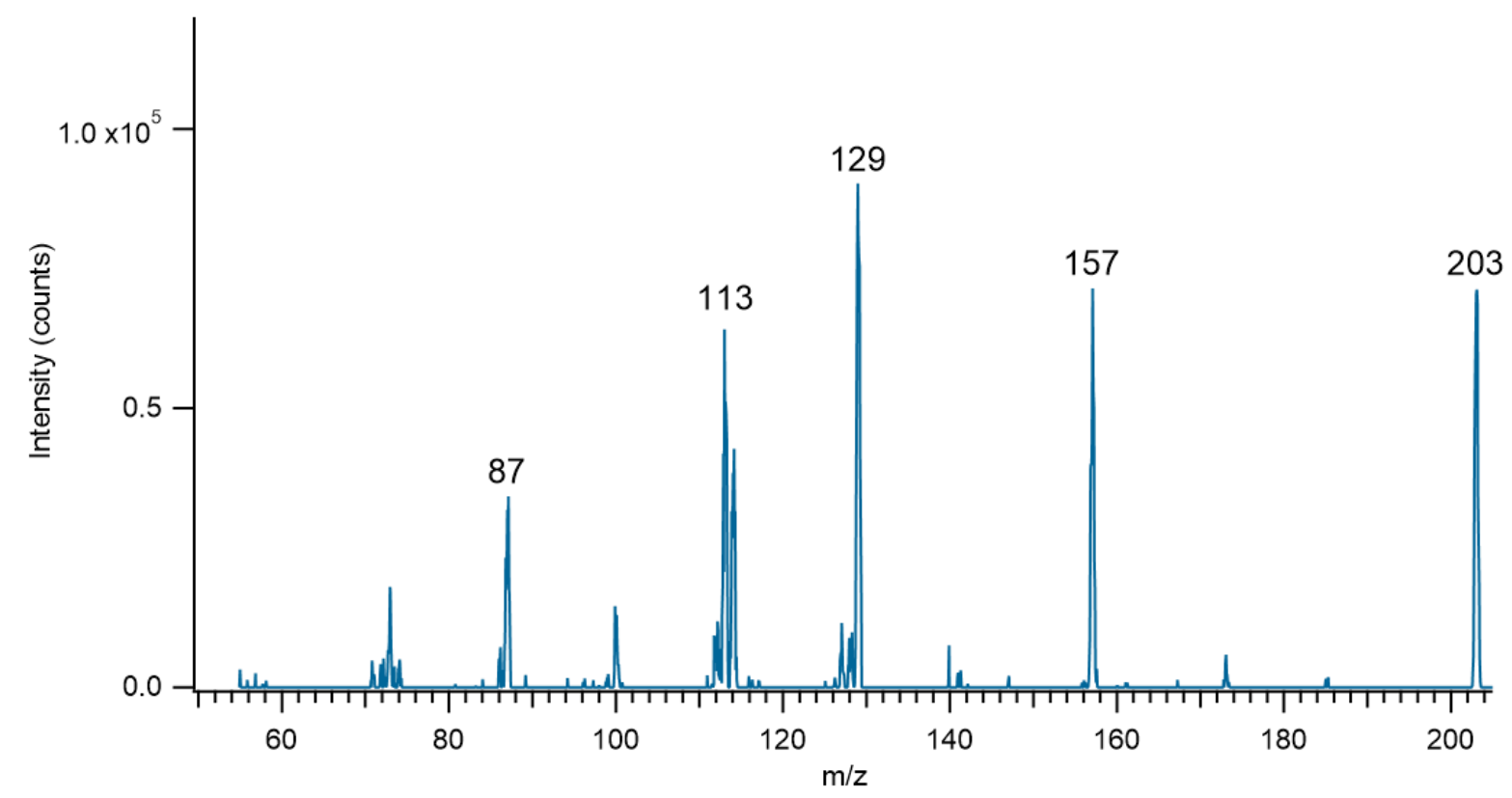

Figure S5: DART MS/MS product scan of $\mathrm{m} / \mathrm{z} 203$, assigned to $D N F$, which shows typical fragmentation patterns seen in the literature for DNF. ${ }^{1-5}$ Spectrum was obtained using $6 \mathrm{eV}$ collision energy on a $0.5 \%$ DNF/KBr pellet. 


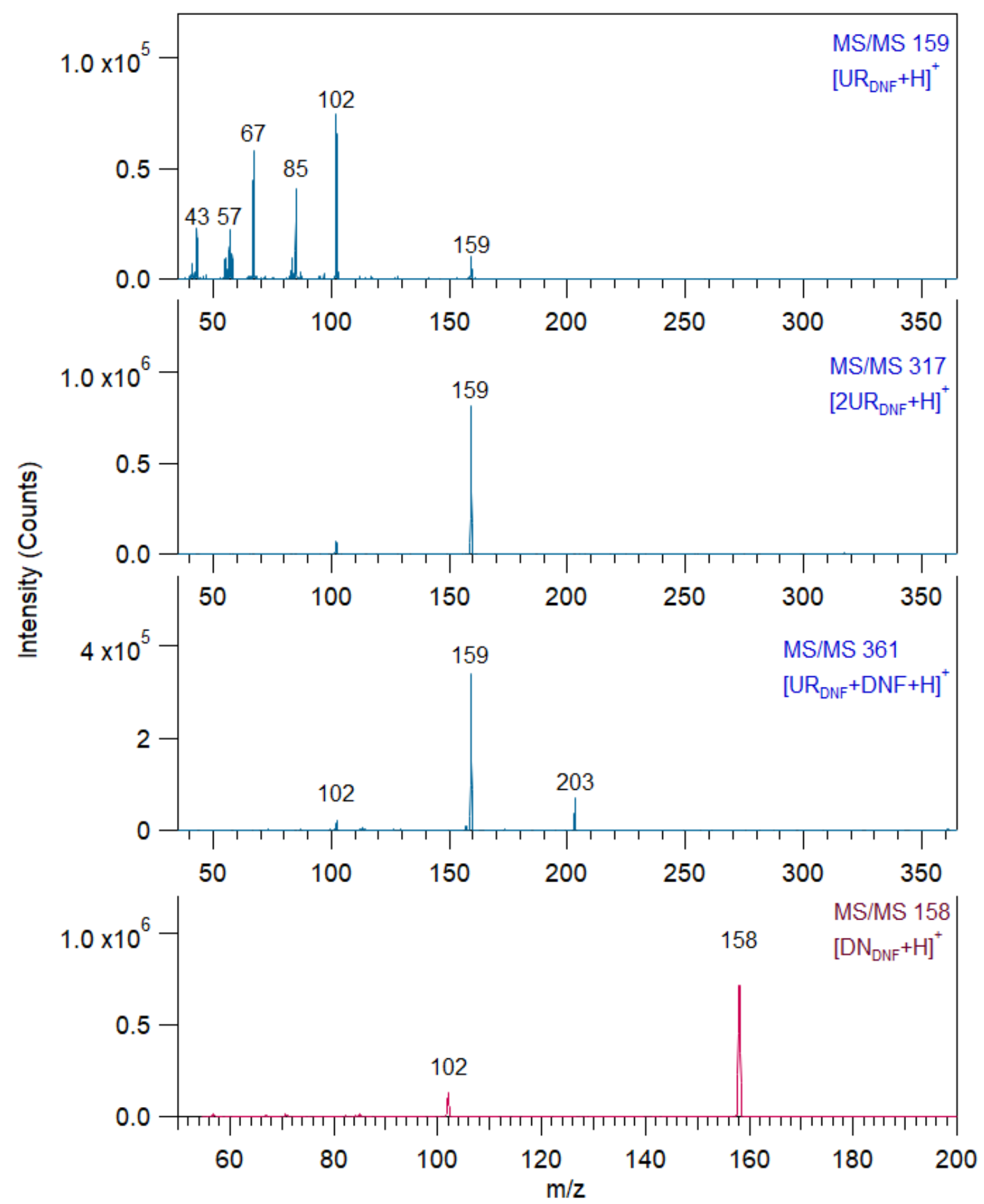

Figure S6: $M S / M S$ product scan of ions assigned to $U R_{D N F}\left(\right.$ blue) and $D N_{D N F}($ red). The ions at $\mathrm{m} / z 158$ and 159 show typical fragmentation patterns seen in the literature, ${ }^{1-3}$ and a fragmentation mechanism is proposed in Scheme S1. The product ion scan of $\mathrm{m} / \mathrm{z} 317$ shows the ion $\mathrm{m} / \mathrm{z} 159$ attributed to $U R_{D N F}$, resulting in the identification as $\left[2 U R_{D N F}+H\right]^{+}$. The product ion scan of $\mathrm{m} / \mathrm{z}, 361$ shows fragments for $U R_{D N F}$ at $\mathrm{m} / \mathrm{z}, 159$ and $D N F(M W 202)$ at $\mathrm{m} / \mathrm{z}$ 203 , resulting in the identification as $\left[U R_{D N F}+D N F+H\right]^{+}$. Samples were obtained using 6-20 eV collision energy on a $2.48 \% \mathrm{DNF} / \mathrm{KBr}$ pellet irradiated at $254 \mathrm{~nm}$ to $38 \%$ loss of DNF. 

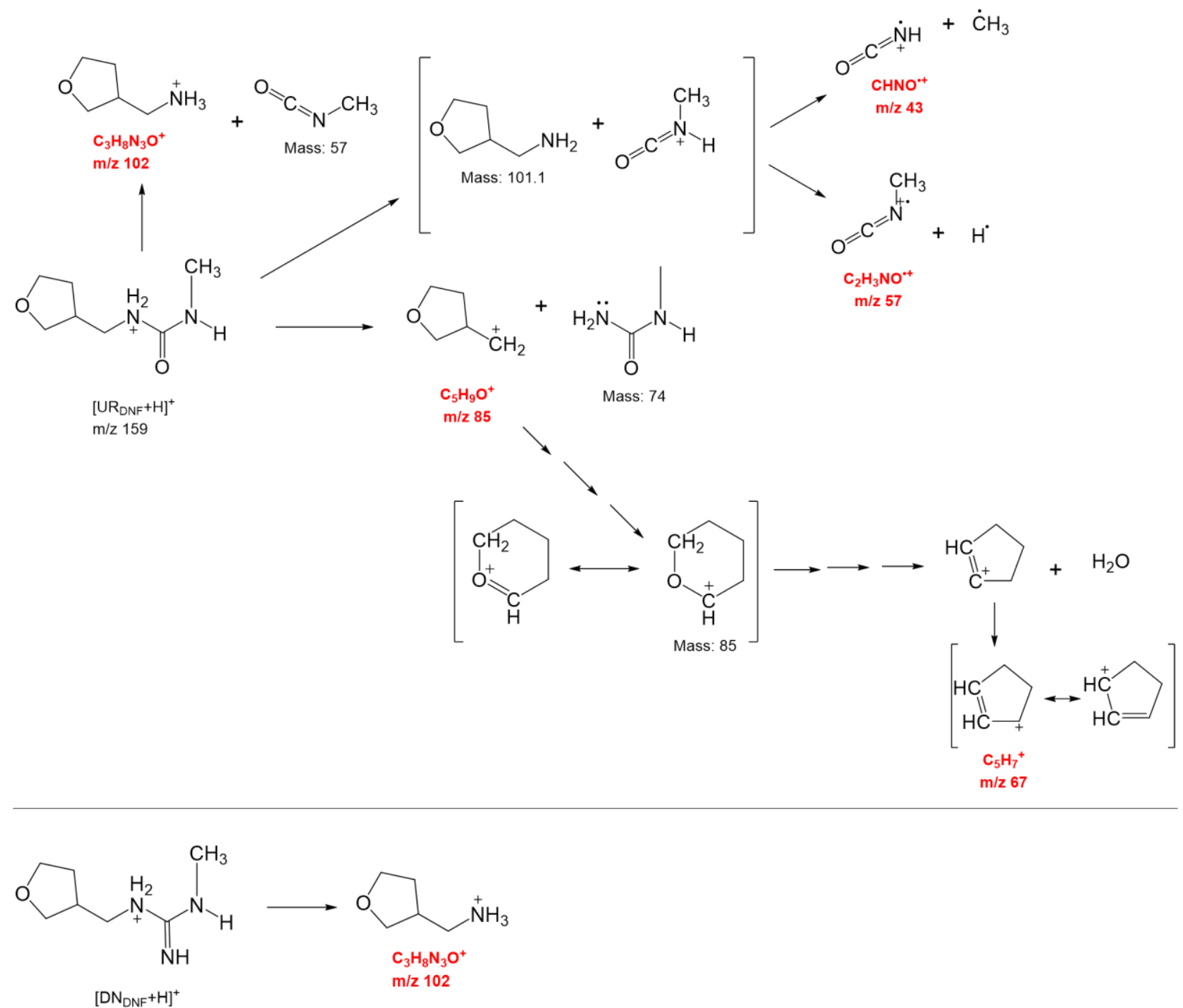

$\mathrm{m} / \mathrm{z} 158$

Scheme S1: Proposed MS/MS fragmentation of DNF degradation products. Red text indicates ions observed in MS/MS product scans in Fig. S6. 


\section{G. HRMS of DNF solutions}

\section{Table S1: HRMS Results}

$\begin{array}{cccc}\text { Observed mass (Da) } & \begin{array}{c}\text { Elemental } \\ \text { Formula }\end{array} & \text { Theoretical mass (Da) } & \text { Absolute mass error (mDa) } \\ 203.1139 & \mathrm{C}_{7} \mathrm{H}_{15} \mathrm{O}_{3} \mathrm{~N}_{4}{ }^{+} & 203.1139 & 0.0 \mathrm{mDa} \\ 172.1081 & \mathrm{C}_{7} \mathrm{H}_{14} \mathrm{O}_{2} \mathrm{~N}_{3}{ }^{+} & 172.1081 & 0.0 \mathrm{mDa} \\ 156.1133 & \mathrm{C}_{7} \mathrm{H}_{14} \mathrm{ON}_{3}{ }^{+} & 156.1132 & -0.1 \mathrm{mDa}\end{array}$

Additional peaks at $m / z 225.0959$ and 427.2021 attributed to $\left[\mathrm{C}_{7} \mathrm{H}_{14} \mathrm{O}_{3} \mathrm{~N}_{4}+\mathrm{Na}\right]^{+}$and $\left[2 \times \mathrm{C}_{7} \mathrm{H}_{14} \mathrm{O}_{3} \mathrm{~N}_{4}+\mathrm{Na}\right]^{+}$adducts were also observed in the UPLC-DAD-RHMS mass spectra (with absolute mass difference of 0.0 and $0.3 \mathrm{mDa}$ respectively from the theoretical mass). All reported data are within the $3 \mathrm{mDa}$ acceptable mass difference defined by the Journal of Organic Chemistry (Greaves and Roboz, 2013). ${ }^{6}$ 
H. Photolysis rate constants $\left(k_{\mathrm{p}}\right)$ using $254 \mathrm{~nm}$ irradiation

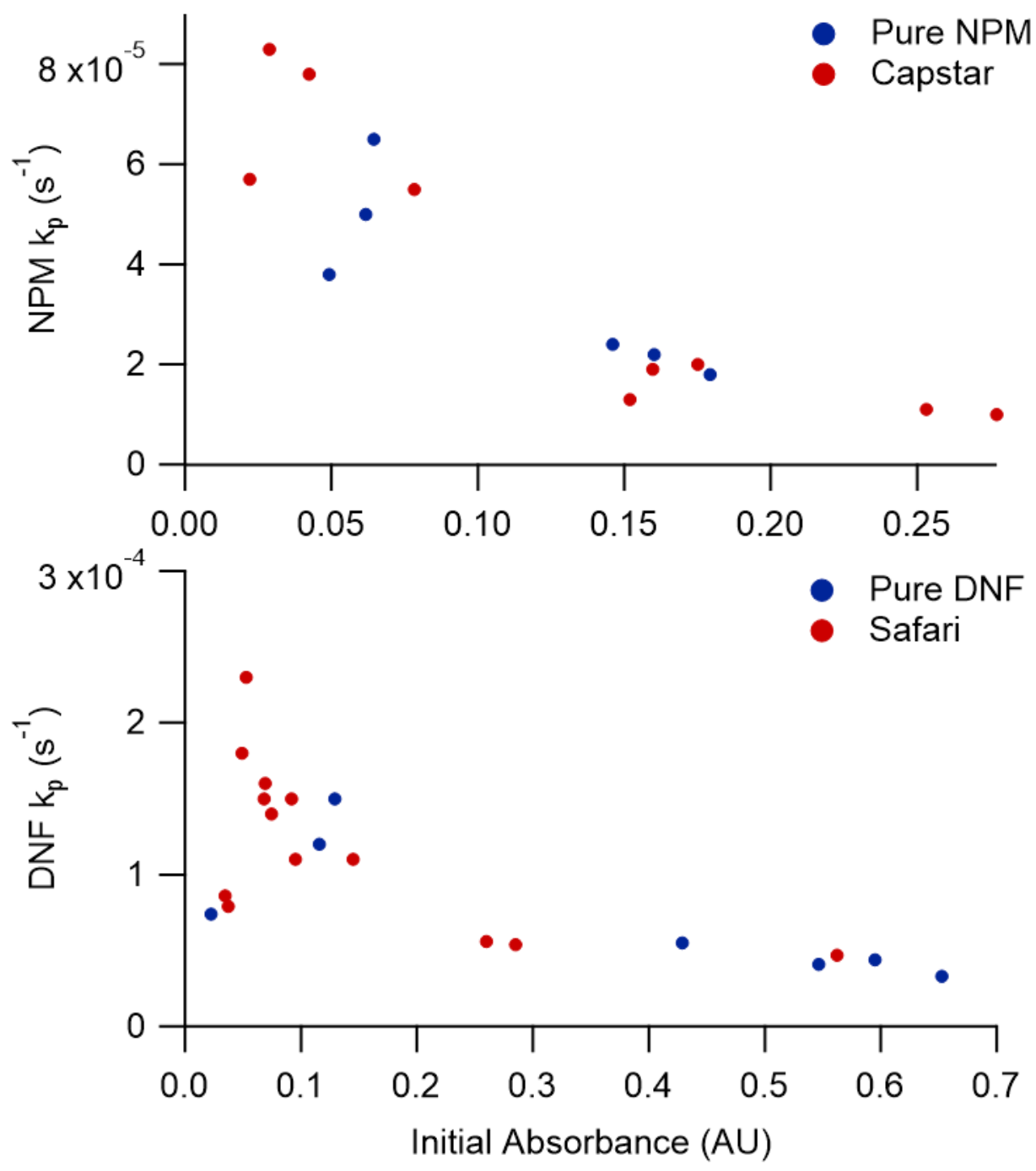

Figure S7: Photolysis rate constants $\left(k_{p}\right)$ at varying initial absorbances (proportional to concentration) recorded for NPM and Capstar ${ }^{\mathrm{TM}}$ (top) and DNF and Safari ${ }^{\circledR}$ (bottom). 


\section{Quantum yield determination via 2NB actinometer}

\section{Absolute Light Intensities}

To calculate quantum yields for loss of the NNs, absolute light intensities for the lamps were measured using 2-nitrobenzaldehyde (2NB) as an actinometer. From 250-430 nm, solid 2NB photochemically converts into 2-nitrosobenzoic acid with a photolysis quantum yield $(\varphi)$ of $0.5 .^{7-10} \mathrm{KBr}$ pellets with known amounts of $2 \mathrm{NB}$ (Fluka, $\geq 99.0 \%$ ) were prepared in the same manner as the NN samples. The $2 \mathrm{NB}$ pellets were then placed in the FTIR cell in $1 \mathrm{~atm} \mathrm{~N}_{2}$ and irradiated using the various lamps. The lamps were removed temporarily at chosen time intervals to allow the IR beam to sample the $\mathrm{KBr}$ pellet, and the lamps were then replaced to obtain the next data point. The removal and replacement of the lamp was done carefully to limit the effect on the placement of the lamp relative to the pellet within the holder. As the pellets do not have a perfectly homogeneous composition, this location adjustment is the major uncertainty that can affect the linearity of the measurements.

The decrease in the 2NB peak at $1353 \mathrm{~cm}^{-1}$ was followed with time in transmission mode. Figure S8a shows the spectrum of $2 \mathrm{NB}$ before photolysis and the difference spectrum after irradiation using the 305 nm broadband lamp.
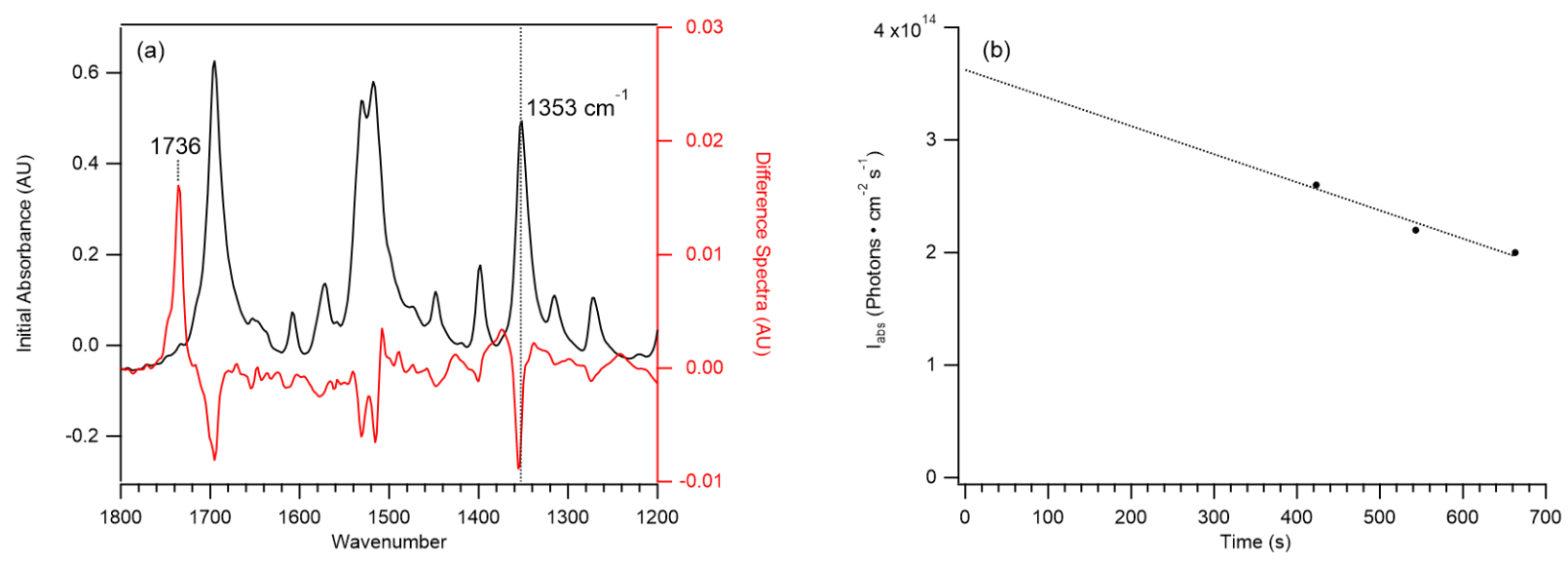

Figure S8 (a) FTIR spectra of $2 \mathrm{NB}$ in $\mathrm{KBr}$ before photolysis (black trace) and the difference spectrum after irradiation using the $305 \mathrm{~nm}$ broadband lamp (red trace). Negative peaks represent loss of $2 \mathrm{NB}$ while positive peaks represent new products formed, seen here at $1736 \mathrm{~cm}^{-1}$ corresponding to the formation of $C=O$ bond in 2-

nitrosobenzoic acid. (b) Absorbed light intensities as a function of exposure time for a typical experiment using the $305 \mathrm{~nm}$ broadband lamp to obtain, by extrapolation, the true absorbed lamp intensity, $I_{a b s, t=0}$.

The relative intensity, $\mathrm{I}_{\mathrm{rel}}(\lambda)$, of the photolysis lamps is related to the absolute light intensity, $\mathrm{I}_{\mathrm{abs}}(\lambda)$ (Fig. S3), through a correction factor, CF, using Eqn. S1:

$$
\begin{gathered}
I_{a b s}(\lambda)=C F \times I_{r e l}(\lambda) \\
\sum I_{a b s}(\lambda)=C F \times \sum I_{r e l}(\lambda)
\end{gathered}
$$

The CF for each lamp was obtained as follows:

(1) the number of $2 \mathrm{NB}$ molecules irradiated is determined using the relative weights of $2 \mathrm{NB}$ and $\mathrm{KBr}$ used to make the mixture and the total mass of the pellet; 
(2) the loss of $2 \mathrm{NB}$ molecules is measured from the change in absorbance at $1353 \mathrm{~cm}^{-1}$ as a function of time $(\Delta 2 \mathrm{NB} / \Delta \mathrm{t})$. With the known quantum yield of 0.5 , this loss is equivalent to half the number of photons absorbed per unit time. It was divided by the pellet surface area to give units of photons $\cdot \mathrm{cm}^{-2} \cdot \mathrm{s}^{-1}$.

(3) the value of $(\Delta 2 \mathrm{NB} / \Delta \mathrm{t})$ decreases with time due to shielding by the products, ${ }^{8}$ so that this rate was extrapolated back to $t=0$ (Fig. S9b) to obtain the initial total photon flux, $I_{a b s, ~} t=0$ (photons

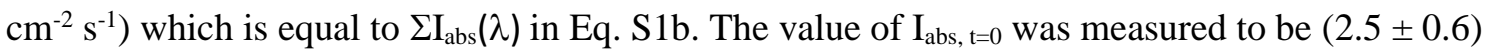
$\times 10^{15}$ photons $\mathrm{cm}^{-2} \mathrm{~s}^{-1}$ for the $254 \mathrm{~nm}$ lamp, $(3.5 \pm 1.2) \times 10^{14}$ photons $\mathrm{cm}^{-2} \mathrm{~s}^{-1}$ for the $305 \mathrm{~nm}$ broadband lamp, and $(5.3 \pm 0.6) \times 10^{14}$ for the $350 \mathrm{~nm}$ broadband lamp.

(4) Using equation $S 1$, the $I_{a b s, ~}=0$ value was divided by $\Sigma I_{\text {rel }}$, which is the sum of the relative light intensity shown in Fig. $\mathbf{S 3}$ across all wavelengths, to determine the CF. The CF was measured to be $(3.2 \pm 0.8) \times 10^{10}$ for the $254 \mathrm{~nm}$ lamp, $(7.2 \pm 2.5) \times 10^{9}$ for the $305 \mathrm{~nm}$ lamp, and $(5.2 \pm 0.6) \times$ $10^{9}$ for the $350 \mathrm{~nm}$ lamp.

\section{Quantum Yield Calculation}

With the CF thus determined, the quantum yield for the NN was calculated from measured photolysis rate constants $\left(k_{p}\right)$ for the NN using Eqn. S2:

$$
k_{p}=\phi_{N N} \cdot \sum \sigma_{N N}(\lambda) I_{r e l}(\lambda) \cdot C F
$$

where $\sigma_{\mathrm{NN}}(\lambda)$ is the absorption cross section of the $\mathrm{NN}$ as a function of wavelength (Fig. 1b) and the photolysis quantum yield $(\phi)$ is assumed to be independent over the wavelength region of the lamp.

Figure S9 shows typical decays for DNF and Safari ${ }^{\circledR}$ during photolysis using the $305 \mathrm{~nm}$ broadband

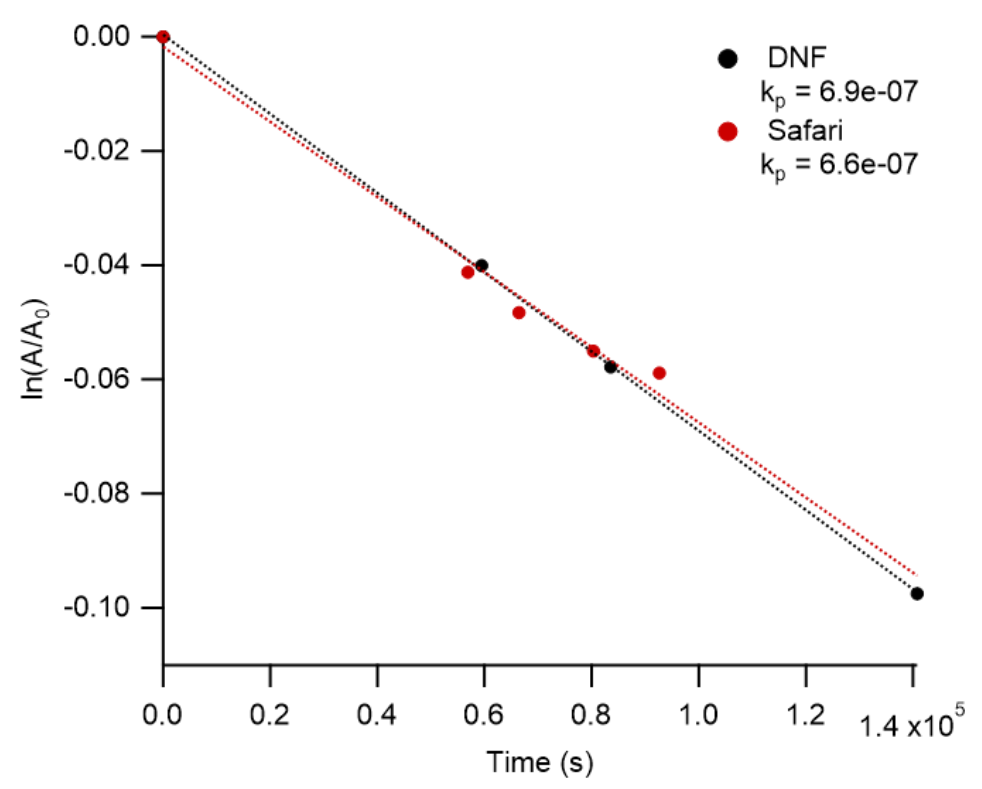

lamp. The values for each experiment used to calculate quantum yields are summarized in Table S2.
Figure S9: First order decays of 0.49\% pure $\mathrm{DNF} / \mathrm{KBr}$ and the formulated counterpart $2.30 \%$ Safari $(0.46 \%$ $\mathrm{DNF} / \mathrm{KBr}$ ) from which photolysis rate constants were obtained. Values for individual runs and average values at each wavelength are given in Table S1. 
Table S2: Photolysis rate constants $\left(k_{p}\right)$ and quantum yields $(\phi)$ for solid $N N$ in $\mathrm{KBr}$ pellets

\begin{tabular}{|c|c|c|c|c|c|}
\hline Lamp & $\mathrm{NN}$ & $\begin{array}{c}\text { Concentration } \\
(\% \mathrm{w} / \mathrm{w})\end{array}$ & $\%$ Loss & $\mathrm{k}_{\mathrm{p}}\left(\mathrm{s}^{-1}\right)^{\mathrm{a}}$ & $\phi^{\mathrm{b}}$ \\
\hline \multirow[t]{4}{*}{254} & DNF & $0.32 \%$ & $67 \%$ & $1.5 \mathrm{E}-04$ & $2.0 \mathrm{E}-03$ \\
\hline & DNF & $0.49 \%$ & $59 \%$ & $1.2 \mathrm{E}-04$ & $1.6 \mathrm{E}-03$ \\
\hline & DNF & $0.18 \%$ & $40 \%$ & 7.4E-05 & $9.5 \mathrm{E}-04$ \\
\hline & & & Average & $(1.2 \pm 0.4) \times 10^{-4}$ & $(1.5 \pm 0.6) \times 10^{-3}$ \\
\hline \multirow[t]{11}{*}{254} & Safari ${ }^{\circledR}$ & $2.20 \%$ & $12 \%$ & $1.1 \mathrm{E}-04$ & $1.5 \mathrm{E}-03$ \\
\hline & Safari ${ }^{\circledR}$ & $2.20 \%$ & $14 \%$ & $1.5 \mathrm{E}-04$ & $2.0 \mathrm{E}-03$ \\
\hline & Safari ${ }^{\circledR}$ & $0.53 \%$ & $47 \%$ & $1.5 \mathrm{E}-04$ & $2.0 \mathrm{E}-03$ \\
\hline & Safari ${ }^{\circledR}$ & $0.53 \%$ & $49 \%$ & $1.8 \mathrm{E}-04$ & 2.3E-03 \\
\hline & Safari ${ }^{\circledR}$ & $0.53 \%$ & $52 \%$ & $1.6 \mathrm{E}-04$ & $2.0 \mathrm{E}-03$ \\
\hline & Safari ${ }^{\circledR}$ & $0.53 \%$ & $50 \%$ & 2.3E-04 & $2.9 \mathrm{E}-03$ \\
\hline & Safari ${ }^{\circledR}$ & $1.09 \%$ & $51 \%$ & $1.4 \mathrm{E}-04$ & $1.9 \mathrm{E}-03$ \\
\hline & Safari ${ }^{\circledR}$ & $1.09 \%$ & $49 \%$ & $1.1 \mathrm{E}-04$ & $1.4 \mathrm{E}-03$ \\
\hline & Safari ${ }^{\circledR}$ & $0.34 \%$ & $50 \%$ & $8.6 \mathrm{E}-05$ & $1.1 \mathrm{E}-03$ \\
\hline & Safari® & $0.34 \%$ & $53 \%$ & 7.9E-05 & $1.0 \mathrm{E}-03$ \\
\hline & & & Average & $(1.4 \pm 0.4) \times 10^{-4}$ & $(1.8 \pm 0.7) \times 10^{-3}$ \\
\hline \multirow[t]{4}{*}{254} & NPM & $0.50 \%$ & $37 \%$ & $6.5 \mathrm{E}-05$ & $1.6 \mathrm{E}-03$ \\
\hline & NPM & $0.19 \%$ & $19 \%$ & $3.8 \mathrm{E}-05$ & $9.2 \mathrm{E}-04$ \\
\hline & NPM & $0.19 \%$ & $21 \%$ & 3.3E-05 & 8.0E-04 \\
\hline & & & Average & $(4.6 \pm 2) \times 10^{-5}$ & $(1.1 \pm 0.5) \times 10^{-3}$ \\
\hline \multirow[t]{5}{*}{254} & Capstar $^{\mathrm{TM}}$ & $5.00 \%$ & $40 \%$ & $5.5 \mathrm{E}-05$ & $1.3 \mathrm{E}-03$ \\
\hline & Capstar ${ }^{\mathrm{TM}}$ & $0.10 \%$ & $61 \%$ & 8.3E-05 & $2.0 \mathrm{E}-03$ \\
\hline & Capstar $^{\mathrm{TM}}$ & $0.10 \%$ & $41 \%$ & $7.8 \mathrm{E}-05$ & $1.9 \mathrm{E}-03$ \\
\hline & Capstar $^{\mathrm{TM}}$ & $0.10 \%$ & $51 \%$ & 5.7E-05 & $1.4 \mathrm{E}-03$ \\
\hline & & & Average & $(6.8 \pm 1) \times 10^{-5}$ & $(1.6 \pm 0.5) \times 10^{-3}$ \\
\hline \multirow[t]{9}{*}{305} & DNF & $0.92 \%$ & $4 \%$ & $5.0 \mathrm{E}-07$ & $3.0 \mathrm{E}-04$ \\
\hline & DNF & $0.91 \%$ & $9 \%$ & 4.9E-07 & $2.9 \mathrm{E}-04$ \\
\hline & DNF & $0.91 \%$ & $7 \%$ & $6.3 \mathrm{E}-07$ & $3.8 \mathrm{E}-04$ \\
\hline & DNF & $0.91 \%$ & $9 \%$ & $6.9 \mathrm{E}-07$ & $4.1 \mathrm{E}-04$ \\
\hline & DNF & $0.91 \%$ & $8 \%$ & $5.8 \mathrm{E}-07$ & $3.5 \mathrm{E}-04$ \\
\hline & DNF & $0.49 \%$ & $49 \%$ & $7.5 \mathrm{E}-07$ & $4.5 \mathrm{E}-04$ \\
\hline & DNF & $0.63 \%$ & $44 \%$ & $5.5 \mathrm{E}-07$ & $3.3 \mathrm{E}-04$ \\
\hline & DNF & $0.58 \%$ & $50 \%$ & 5.6E-07 & 3.3E-04 \\
\hline & & & Average & $(5.9 \pm 0.9) \times 10^{-7}$ & $(3.5 \pm 1.4) \times 10^{-4}$ \\
\hline 305 & Safari ${ }^{\circledR}$ & $4.66 \%$ & $6 \%$ & $6.6 \mathrm{E}-07$ & $3.9 \mathrm{E}-04$ \\
\hline
\end{tabular}




\begin{tabular}{|c|c|c|c|c|c|}
\hline & Safari® & $4.62 \%$ & $9 \%$ & $5.4 \mathrm{E}-07$ & $3.2 \mathrm{E}-04$ \\
\hline & Safari® ${ }^{\circledR}$ & $4.62 \%$ & $5 \%$ & 3.7E-07 & 2.2E-04 \\
\hline & Safari ${ }^{\circledR}$ & $4.62 \%$ & $14 \%$ & $9.0 \mathrm{E}-07$ & $5.4 \mathrm{E}-04$ \\
\hline & Safari ${ }^{\circledR}$ & $4.62 \%$ & $11 \%$ & $1.0 \mathrm{E}-06$ & $6.1 \mathrm{E}-04$ \\
\hline & Safari ${ }^{\circledR}$ & $0.10 \%$ & $17 \%$ & 5.3E-07 & $3.2 \mathrm{E}-04$ \\
\hline & Safari ${ }^{\circledR}$ & $2.30 \%$ & $31 \%$ & $4.0 \mathrm{E}-07$ & $2.4 \mathrm{E}-04$ \\
\hline & Safari® & $2.30 \%$ & $26 \%$ & 5.9E-07 & 3.5E-04 \\
\hline & & & Average & $(6.3 \pm 2) \times 10^{-7}$ & $(3.7 \pm 1.9) \times 10^{-4}$ \\
\hline \multirow[t]{6}{*}{305} & NPM & $0.50 \%$ & $6 \%$ & $1.8 \mathrm{E}-06$ & $2.5 \mathrm{E}-04$ \\
\hline & NPM & $0.50 \%$ & $3 \%$ & $2.2 \mathrm{E}-06$ & $3.0 \mathrm{E}-04$ \\
\hline & NPM & $0.50 \%$ & $4 \%$ & $1.9 \mathrm{E}-06$ & $2.6 \mathrm{E}-04$ \\
\hline & NPM & $0.50 \%$ & $62 \%$ & 8.9E-07 & $1.2 \mathrm{E}-04$ \\
\hline & NPM & $0.50 \%$ & $47 \%$ & 8.8E-07 & $1.2 \mathrm{E}-04$ \\
\hline & & & Average & $(1.5 \pm 0.6) \times 10^{-6}$ & $(2.1 \pm 1.1) \times 10^{-4}$ \\
\hline \multirow[t]{8}{*}{305} & Capstar ${ }^{\mathrm{TM}}$ & $5.00 \%$ & $5 \%$ & $1.8 \mathrm{E}-06$ & $2.5 \mathrm{E}-04$ \\
\hline & Capstar $^{\mathrm{TM}}$ & $4.97 \%$ & $2 \%$ & $1.3 \mathrm{E}-06$ & $1.8 \mathrm{E}-04$ \\
\hline & Capstar ${ }^{\mathrm{TM}}$ & $4.97 \%$ & $4 \%$ & $1.7 \mathrm{E}-06$ & $2.3 \mathrm{E}-04$ \\
\hline & Capstar ${ }^{\mathrm{TM}}$ & $4.97 \%$ & $3 \%$ & $1.4 \mathrm{E}-06$ & $1.9 \mathrm{E}-04$ \\
\hline & Capstar ${ }^{\mathrm{TM}}$ & $4.95 \%$ & $2 \%$ & $1.6 \mathrm{E}-06$ & $2.2 \mathrm{E}-04$ \\
\hline & Capstar $^{\mathrm{TM}}$ & $4.95 \%$ & $14 \%$ & $1.4 \mathrm{E}-06$ & 1.9E-04 \\
\hline & Capstar $^{\mathrm{TM}}$ & $4.95 \%$ & $48 \%$ & $1.6 \mathrm{E}-06$ & $2.2 \mathrm{E}-04$ \\
\hline & & & Average & $(1.5 \pm 0.2) \times 10^{-6}$ & $(2.1 \pm 0.8) \times 10^{-4}$ \\
\hline \multirow[t]{5}{*}{350} & NPM & $0.48 \%$ & $11 \%$ & $4.0 \mathrm{E}-06$ & $1.8 \mathrm{E}-04$ \\
\hline & NPM & $0.48 \%$ & $11 \%$ & $6.4 \mathrm{E}-06$ & $2.9 \mathrm{E}-04$ \\
\hline & NPM & $0.48 \%$ & $8 \%$ & $3.7 \mathrm{E}-06$ & $1.7 \mathrm{E}-04$ \\
\hline & NPM & $0.48 \%$ & $7 \%$ & 4.0E-06 & $1.8 \mathrm{E}-04$ \\
\hline & & & Average & $(4.5 \pm 1.3) \times 10^{-6}$ & $(2.0 \pm 0.6) \times 10^{-4}$ \\
\hline \multirow[t]{4}{*}{350} & Capstar ${ }^{\mathrm{TM}}$ & $4.80 \%$ & $7 \%$ & $2.7 \mathrm{E}-06$ & $1.2 \mathrm{E}-04$ \\
\hline & Capstar ${ }^{\mathrm{TM}}$ & $4.80 \%$ & $3 \%$ & $1.6 \mathrm{E}-06$ & $7.0 \mathrm{E}-05$ \\
\hline & Capstar ${ }^{\mathrm{TM}}$ & $4.80 \%$ & $4 \%$ & $1.3 \mathrm{E}-06$ & $6.0 \mathrm{E}-05$ \\
\hline & & & Average & $(1.9 \pm 0.8) \times 10^{-6}$ & $(0.84 \pm 0.4) \times 10^{-4}$ \\
\hline
\end{tabular}

${ }^{\text {a }}$ Standard deviation is $1 \sigma$, calculated as $\sigma=\left(\frac{\sum\left(x_{i}-\mu\right)^{2}}{N}\right)^{1 / 2}$

${ }^{\mathrm{b}}$ Standard deviation $(1 \sigma \phi)$ is determined by uncertainties in the NN photolysis rate constants $(\mathrm{kNN})$ and the measurement of the intensity correction factor (CF) from the 2NB photolysis rate constant (k2NB), calculated as: $: \frac{\sigma_{\phi}}{\phi}=\left[\left(\frac{\sigma_{k p}}{k p}\right)^{2}+\left(\frac{\sigma_{C F}}{C F}\right)^{2}\right]^{\frac{1}{2}}$ where uncertainties in $\Sigma \sigma \mathrm{I}$ are taken to be negligible compared to those in $\mathrm{k}_{\mathrm{p}}$ and $\mathrm{CF}$. 


\section{References}

(1) Raina-Fulton, R.; Behdarvandan, A., Liquid chromatography-mass spectrometry for the determination of neonicotinoid insecticides and their metabolites in biological, environmental and food commodity matrices. Trends in Chromatography 2016, 10, 51-79.

(2) Kamel, A., Refined methodology for the determination of neonicotinoid pesticides and their metabolites in honey bees and bee products by liquid chromatography-tandem mass spectrometry (LCMS/MS). J Agr Food Chem 2010, 58 (10), 5926-5931.

(3) Yang, Z. H.; Zhang, K. K.; Chen, L. Z.; Liu, B.; Zhang, Q. T.; Zhang, H. Z.; Sun, C. Y.; Hu, D. Y., Determination of dinotefuran and its metabolites in orange pulp, orange peel, and whole orange using liquid chromatography-tandem mass spectrometry. J. AOAC Int. 2017, 100 (5), 1551-1558.

(4) Xie, W.; Han, C.; Qian, Y.; Ding, H. Y.; Chen, X. M.; Xi, J. Y., Determination of neonicotinoid pesticides residues in agricultural samples by solid-phase extraction combined with liquid chromatography-tandem mass spectrometry. J. Chromatogr. A 2011, 1218 (28), 4426-4433.

(5) Kamel, A.; Qian, Y. R.; Kolbe, E.; Stafford, C., Development and validation of a multiresidue method for the determination of neonicotinoid and macrocyclic lactone pesticide residues in milk, fruits, and vegetables by ultra-performance liquid chromatography/MS/MS. J. AOAC Int. 2010, 93 (2), 389-399.

(6) Greaves, J. L.; Roboz, J., Mass Spectrometry for Novices. Taylor and Frances: Boca Raton, FL, 2013.

(7) Leighton, P. A.; Lucy, F. A., The photoisomerization of the o-nitrobenzaldehydes I. photochemical results. J. Chem. Phys. 1934, 2 (11), 756-759.

(8) Pitts, J. N.; Wan, J. K. S.; Schuck, E., Photochemical studies in an alkali halide matrix. I. An onitrobenzaldehyde actinometer and its application to a kinetic study of the photoreduction of benzophenone by benzhydrol in a pressed potassium bromide disk. J. Am. Chem. Soc. 1964, 86 (18), 3606-3610.

(9) Vichutinskaya, E. V.; Postnikov, L. M.; Kushnerev, M. Y., Use of ortho-nitrobenzaldehyde as an internal actinometer in investigation of photooxidative degradation of thin unoriented polycaproamide films. Vysokomol Soedin a+ 1975, 17 (3), 621-625.

(10) Allen, J. M.; Allen, S. K.; Baertschi, S. W., 2-Nitrobenzaldehyde: a convenient UV-A and UV-B chemical actinometer for drug photostability testing. J. Pharm. Biomed. Anal. 2000, 24 (2), 167-178. 